\title{
A note on truncations in fractional Sobolev spaces
}

\author{
Roberta Musina*, $^{*}$ and Alexander I. Nazarov ${ }^{\dagger, \ddagger}, \boldsymbol{\uparrow}$ \\ *Dipartimento di Scienze Matematiche \\ Informatiche e Fisiche, Università di Udine \\ via delle Scienze 206, 33100 Udine, Italy \\ ${ }^{\dagger}$ St.Petersburg Department of Steklov Institute \\ Fontanka, 27, St.Petersburg, 191023, Russia \\ $¥$ St.Petersburg State University \\ Universitetskii pr. 28, St.Petersburg, 198504, Russia \\ \$roberta.musina@uniud.it \\ 『al.il.nazarov@gmail.com \\ Received 19 January 2017 \\ Accepted 16 June 2017 \\ Published 13 March 2019 \\ Communicated by Ari Laptev
}

\begin{abstract}
We study the Nemytskii operators $u \mapsto|u|$ and $u \mapsto u^{ \pm}$in fractional Sobolev spaces $H^{s}\left(\mathbb{R}^{n}\right), s>1$.
\end{abstract}

Keywords: Fractional Laplacian; Sobolev spaces; truncation operators.

Mathematics Subject Classification: 46E35, 47H30

\section{Introduction: Main Result}

In this paper, we discuss the relation between the map $u \mapsto|u|$ and the Dirichlet Laplacian. Recall that the Dirichlet Laplacian $\left(-\Delta_{\mathbb{R}^{n}}\right)^{s} u$ of order $s>0$ of a function $u \in L^{2}\left(\mathbb{R}^{n}\right), n \geq 1$, is the distribution

$$
\left\langle\left(-\Delta_{\mathbb{R}^{n}}\right)^{s} u, \varphi\right\rangle \equiv \int_{\mathbb{R}^{n}} u\left(-\Delta_{\mathbb{R}^{n}}\right)^{s} \varphi d x:=\int_{\mathbb{R}^{n}}|\xi|^{2 s} \mathcal{F}[\varphi] \overline{\mathcal{F}[u]} d \xi, \quad \varphi \in \mathcal{C}_{0}^{\infty}\left(\mathbb{R}^{n}\right),
$$

where

$$
\mathcal{F}[u](\xi)=(2 \pi)^{-\frac{n}{2}} \int_{\mathbb{R}^{n}} e^{-i \xi \cdot x} u(x) d x
$$

This is an Open Access article published by World Scientific Publishing Company. It is distributed under the terms of the Creative Commons Attribution 4.0 (CC-BY) License. Further distribution of this work is permitted, provided the original work is properly cited. 
is the Fourier transform in $\mathbb{R}^{n}$. The Sobolev-Slobodetskii space

$$
H^{s}\left(\mathbb{R}^{n}\right)=\left\{u \in L^{2}\left(\mathbb{R}^{n}\right) \mid\left(-\Delta_{\mathbb{R}^{n}}\right)^{\frac{s}{2}} u \in L^{2}\left(\mathbb{R}^{n}\right)\right\}
$$

naturally inherits a Hilbertian structure from the scalar product

$$
(u, v)=\left\langle\left(-\Delta_{\mathbb{R}^{n}}\right)^{s} u, v\right\rangle+\int_{\mathbb{R}^{n}} u v d x .
$$

The standard reference for the operator $\left(-\Delta_{\mathbb{R}^{n}}\right)^{s}$ and functions in $H^{s}\left(\mathbb{R}^{n}\right)$ is the monograph [8] by Triebel.

For any positive order $s \notin \mathbb{N}$ we introduce the constant

$$
C_{n, s}=\frac{2^{2 s} s}{\pi^{\frac{n}{2}}} \frac{\Gamma\left(\frac{n}{2}+s\right)}{\Gamma(1-s)} .
$$

Note that

$$
C_{n, s}>0 \text { if }\lfloor s\rfloor \text { is even; } \quad C_{n, s}<0 \text { if }\lfloor s\rfloor \text { is odd, }
$$

where $\lfloor s\rfloor$ stands for the integer part of $s$. It is well known that for $s \in(0,1)$ and $u, v \in H^{s}\left(\mathbb{R}^{n}\right)$ one has

$$
\left\langle\left(-\Delta_{\mathbb{R}^{n}}\right)^{s} u, v\right\rangle=\frac{C_{n, s}}{2} \iint_{\mathbb{R}^{n} \times \mathbb{R}^{n}} \frac{(u(x)-u(y))(v(x)-v(y))}{|x-y|^{n+2 s}} d x d y .
$$

Let us recall some known facts about the Nemytskii operator $|\cdot|: u \mapsto|u|$.

1. $|\cdot|$ is a Lipschitz transform of $H^{0}\left(\mathbb{R}^{n}\right) \equiv L^{2}\left(\mathbb{R}^{n}\right)$ into itself.

2. Let $0<s \leq 1$. Then $|\cdot|$ is a continuous transform of $H^{s}\left(\mathbb{R}^{n}\right)$ into itself, by general results about Nemytskii operators in Sobolev/Besov spaces, see [7, Theorem 5.5.2/3]. Also it is obvious that for $u \in H^{1}\left(\mathbb{R}^{n}\right)$

$$
\langle-\Delta|u|,|u|\rangle=\langle-\Delta u, u\rangle=\int_{\mathbb{R}^{n}}|\nabla u|^{2} d x, \quad\left\langle-\Delta u^{+}, u^{-}\right\rangle=\int_{\mathbb{R}^{n}} \nabla u^{+} \cdot \nabla u^{-} d x=0 .
$$

Here and elsewhere $u^{ \pm}=\max \{ \pm u, 0\}=\frac{1}{2}(|u| \pm u)$, so that $u=u^{+}-u^{-},|u|=$ $u^{+}+u^{-}$. On the other hand, for $s \in(0,1)$ and $u \in H^{s}\left(\mathbb{R}^{n}\right)$ formula (3) gives

$$
\left\langle\left(-\Delta_{\mathbb{R}^{n}}\right)^{s} u^{+}, u^{-}\right\rangle=-C_{n, s} \iint_{\mathbb{R}^{n} \times \mathbb{R}^{n}} \frac{u^{+}(x) u^{-}(y)}{|x-y|^{n+2 s}} d x d y .
$$

From (44) we infer by the polarization identity

$$
4\left\langle\left(-\Delta_{\mathbb{R}^{n}}\right)^{s} u^{+}, u^{-}\right\rangle=\left\langle\left(-\Delta_{\mathbb{R}^{n}}\right)^{s}|u|,|u|\right\rangle-\left\langle\left(-\Delta_{\mathbb{R}^{n}}\right)^{s} u, u\right\rangle
$$

that if $u$ changes sign then

$$
\left\langle\left(-\Delta_{\mathbb{R}^{n}}\right)^{s}|u|,|u|\right\rangle<\left\langle\left(-\Delta_{\mathbb{R}^{n}}\right)^{s} u, u\right\rangle, \quad s \in(0,1) .
$$

We also mention [4, Theorem 6] for a different proof and explanation of (5), that includes the case when $\left(-\Delta_{\mathbb{R}^{n}}\right)^{s}$ is replaced by the Navier (or spectral Dirichlet) Laplacian on a bounded Lipschitz domain $\Omega \subset \mathbb{R}^{n}$. 
3. Let $1<s<\frac{3}{2}$. The results in [2, 6] (see also Sec. 4 of the exhaustive survey [3]) imply that $|\cdot|$ is a bounded transform of $H^{s}\left(\mathbb{R}^{n}\right)$ into itself. That is, there exists a constant $c(n, s)$ such that

$$
\left\langle\left(-\Delta_{\mathbb{R}^{n}}\right)^{s}|u|,|u|\right\rangle \leq c(n, s)\left\langle\left(-\Delta_{\mathbb{R}^{n}}\right)^{s} u, u\right\rangle, \quad u \in H^{s}\left(\mathbb{R}^{n}\right) .
$$

In particular, $|\cdot|$ is continuous at $0 \in H^{s}\left(\mathbb{R}^{n}\right)$.

It is easy to show that the assumption $s<\frac{3}{2}$ cannot be improved, see Example 1 below and [2] Proposition, p. 357], where a more general setting involving Besov spaces $B_{p}^{s, q}\left(\mathbb{R}^{n}\right), s \geq 1+\frac{1}{p}$, is considered.

At our knowledge, the continuity of $|\cdot|: H^{s}\left(\mathbb{R}^{n}\right) \rightarrow H^{s}\left(\mathbb{R}^{n}\right), s \in\left(1, \frac{3}{2}\right)$, is an open problem. We can only point out the next simple result.

Proposition 1. Let $0<\tau<s<\frac{3}{2}$. Then $|\cdot|: H^{s}\left(\mathbb{R}^{n}\right) \rightarrow H^{\tau}\left(\mathbb{R}^{n}\right)$ is continuous.

Proof. Recall that $H^{s}\left(\mathbb{R}^{n}\right) \hookrightarrow H^{\tau}\left(\mathbb{R}^{n}\right)$ for $0<\tau<s$. Actually, the Hölder inequality readily gives the well-known interpolation inequality

$$
\begin{aligned}
\left\langle\left(-\Delta_{\mathbb{R}^{n}}\right)^{\tau} v, v\right\rangle & =\int_{\mathbb{R}^{n}}|\xi|^{2 \tau}|\mathcal{F}[v]|^{2} d \xi \\
& \leq\left(\left\langle\left(-\Delta_{\mathbb{R}^{n}}\right)^{s} v, v\right\rangle\right)^{\frac{\tau}{s}}\left(\int_{\mathbb{R}^{n}}|v|^{2} d x\right)^{\frac{s-\tau}{s}}, \quad v \in H^{s}\left(\mathbb{R}^{n}\right) .
\end{aligned}
$$

Since $|\cdot|$ is continuous $L^{2}\left(\mathbb{R}^{n}\right) \rightarrow L^{2}\left(\mathbb{R}^{n}\right)$ and bounded $H^{s}\left(\mathbb{R}^{n}\right) \rightarrow H^{s}\left(\mathbb{R}^{n}\right)$, the statement follows immediately.

Now we formulate our main result. It provides the complete proof of [5, Theorem 1] for $s$ below the threshold $\frac{3}{2}$ and gives a positive answer to a question raised in [1, Remark 4.2] by Abatangelo, Jahors and Saldaña.

Theorem 1. Let $s \in\left(1, \frac{3}{2}\right)$ and $u \in H^{s}\left(\mathbb{R}^{n}\right)$. Then formula (4) holds. In particular, if $u$ changes sign then

$$
\left\langle\left(-\Delta_{\mathbb{R}^{n}}\right)^{s}|u|,|u|\right\rangle>\left\langle\left(-\Delta_{\mathbb{R}^{n}}\right)^{s} u, u\right\rangle .
$$

Our proof is deeply based on the continuity result in Proposition 1 The knowledge of the continuity of $|\cdot|: H^{s}\left(\mathbb{R}^{n}\right) \rightarrow H^{s}\left(\mathbb{R}^{n}\right)$ could considerably simplify it.

We denote by $c$ any positive constant whose value is not important for our purposes. Its value may change line to line. The dependence of $c$ on certain parameters is shown in parentheses.

\section{Preliminary Results and Proof of Theorem 1}

We begin with a simple but crucial identity that has been independently pointed out in [5. Lemma 1] and [1, Lemma 3.11] (without exact value of the constant). Note that it holds for general fractional orders $s>0$. 
Theorem 2. Let $s>0, s \notin \mathbb{N}$. Assume that $v, w \in H^{s}\left(\mathbb{R}^{n}\right)$ have compact and disjoint supports. Then

$$
\left\langle\left(-\Delta_{\mathbb{R}^{n}}\right)^{s} v, w\right\rangle=-C_{n, s} \iint_{\mathbb{R}^{n} \times \mathbb{R}^{n}} \frac{v(x) w(y)}{|x-y|^{n+2 s}} d x d y .
$$

Proof. Let $\rho_{h}$ be a sequence of mollifiers, and put $w_{h}:=w * \rho_{h}$. Formula (3) gives

$$
\begin{aligned}
& \left\langle\left(-\Delta_{\mathbb{R}^{n}}\right)^{s} v, w_{h}\right\rangle \\
& \quad=\left\langle\left(-\Delta_{\mathbb{R}^{n}}\right)^{s-\lfloor s\rfloor} v,(-\Delta)^{\lfloor s\rfloor} w_{h}\right\rangle \\
& \quad=\frac{C_{n, s-\lfloor s\rfloor}}{2} \iint_{\mathbb{R}^{n} \times \mathbb{R}^{n}} \frac{(v(x)-v(y))\left((-\Delta)^{\lfloor s\rfloor} w_{h}(x)-(-\Delta)^{\lfloor s\rfloor} w_{h}(y)\right)}{|x-y|^{n+2(s-\lfloor s\rfloor)}} d x d y .
\end{aligned}
$$

Since for large $h$ the supports of $v$ and $w_{h}$ are separated, we have

$$
\left\langle\left(-\Delta_{\mathbb{R}^{n}}\right)^{s} v, w_{h}\right\rangle=-C_{n, s-\lfloor s\rfloor} \iint_{\mathbb{R}^{n} \times \mathbb{R}^{n}} \frac{v(x)(-\Delta)^{\lfloor s\rfloor} w_{h}(y)}{|x-y|^{n+2(s-\lfloor s\rfloor)}} d y d x .
$$

Here we can integrate by parts. Using (II) one computes for $a>0$

$$
\Delta \frac{C_{n, a}}{|x-y|^{n+2 a}}=\frac{C_{n, a}(n+2 a)(2 a+2)}{|x-y|^{n+2 a+2}}=-\frac{C_{n, a+1}}{|x-y|^{n+2(a+1)}}
$$

and obtains (6) with $w_{h}$ instead of $w$.

Since the supports of $v$ and $w$ are separated, it is easy to pass to the limit as $h \rightarrow \infty$ and to conclude the proof.

Remark 1. Motivated by (6) and (2), Nazarov conjectured in [5] that

$$
\begin{aligned}
& \left\langle\left(-\Delta_{\mathbb{R}^{n}}\right)^{s}|u|,|u|\right\rangle-\left\langle\left(-\Delta_{\mathbb{R}^{n}}\right)^{s} u, u\right\rangle<0 \text { if }\lfloor s\rfloor \text { is even; } \\
& \left\langle\left(-\Delta_{\mathbb{R}^{n}}\right)^{s}|u|,|u|\right\rangle-\left\langle\left(-\Delta_{\mathbb{R}^{n}}\right)^{s} u, u\right\rangle>0 \text { if }\lfloor s\rfloor \text { is odd }
\end{aligned}
$$

for any not integer exponent $s>0$ and for any changing sign function $u \in H^{s}\left(\mathbb{R}^{n}\right)$ such that $u^{ \pm} \in H^{s}\left(\mathbb{R}^{n}\right)$.

Lemma 1. Let $s \in\left(1, \frac{3}{2}\right)$ and $\varepsilon>0$. If a function $u \in H^{s}\left(\mathbb{R}^{n}\right)$ has compact support then $(u-\varepsilon)^{+} \in H^{s}\left(\mathbb{R}^{n}\right)$, and

$$
\left\langle\left(-\Delta_{\mathbb{R}^{n}}\right)^{s}(u-\varepsilon)^{+},(u-\varepsilon)^{+}\right\rangle \leq c(n, s)\left\langle\left(-\Delta_{\mathbb{R}^{n}}\right)^{s} u, u\right\rangle+c(n, s, \operatorname{supp}(u)) \varepsilon^{2} .
$$

Proof. Take a nonnegative function $\eta \in \mathcal{C}_{0}^{\infty}\left(\mathbb{R}^{n}\right)$ such that $\eta \equiv 1$ on $\operatorname{supp}(u)$. Clearly $u-\varepsilon \eta \in H^{s}\left(\mathbb{R}^{n}\right)$. Hence, by Item 3 in the Introduction we have that $(u-\varepsilon \eta)^{+}=(u-\varepsilon)^{+} \in H^{s}\left(\mathbb{R}^{n}\right)$ and

$$
\begin{aligned}
\left\langle\left(-\Delta_{\mathbb{R}^{n}}\right)^{s}(u-\varepsilon)^{+},(u-\varepsilon)^{+}\right\rangle & \leq c(n, s)\left\langle\left(-\Delta_{\mathbb{R}^{n}}\right)^{s}(u-\varepsilon \eta), u-\varepsilon \eta\right\rangle \\
& \leq c(n, s)\left(\left\langle\left(-\Delta_{\mathbb{R}^{n}}\right)^{s} u, u\right\rangle+\varepsilon^{2}\left\langle\left(-\Delta_{\mathbb{R}^{n}}\right)^{s} \eta, \eta\right\rangle\right) .
\end{aligned}
$$

The proof is complete. 
In order to simplify notation, for $u: \mathbb{R}^{n} \rightarrow \mathbb{R}$ and $s>0$ we put

$$
\Phi_{u}^{s}(x, y)=\frac{u^{+}(x) u^{-}(y)}{|x-y|^{n+2 s}} .
$$

Lemma 2. Let $s \in\left(1, \frac{3}{2}\right)$ and $u \in H^{s}\left(\mathbb{R}^{n}\right) \cap \mathcal{C}_{0}^{0}\left(\mathbb{R}^{n}\right)$. Then (4) holds, and in particular $\Phi_{u}^{s} \in L^{1}\left(\mathbb{R}^{n} \times \mathbb{R}^{n}\right)$.

Proof. Thanks to Lemma 1 we have that $\left(u^{-}-\varepsilon\right)^{+} \in H^{s}\left(\mathbb{R}^{n}\right) \cap \mathcal{C}_{0}^{0}\left(\mathbb{R}^{n}\right)$ for any $\varepsilon>0$. Next, the supports of the functions $u^{+}$and $\left(u^{-}-\varepsilon\right)^{+}$are compact and disjoint. Thus we can apply Theorem 2 to get

$$
\left\langle\left(-\Delta_{\mathbb{R}^{n}}\right)^{s} u^{+},\left(u^{-}-\varepsilon\right)^{+}\right\rangle=-C_{n, s} \iint_{\mathbb{R}^{n} \times \mathbb{R}^{n}} \frac{u^{+}(x)\left(u(y)^{-}-\varepsilon\right)^{+}}{|x-y|^{n+2 s}} d x d y .
$$

Take a decreasing sequence $\varepsilon \searrow 0$. From Lemma 1 we infer that $\left(u^{-}-\varepsilon\right)^{+} \rightarrow u^{-}$ weakly in $H^{s}\left(\mathbb{R}^{n}\right)$, as $\left(u^{-}-\varepsilon\right)^{+} \rightarrow u^{-}$in $L^{2}\left(\mathbb{R}^{n}\right)$. Hence the duality product in (7) converges to the duality product in (44). Next, the integrand in the righthand side of (7) increases to $\Phi_{u}^{s}$ a.e. on $\mathbb{R}^{n} \times \mathbb{R}^{n}$. By the monotone convergence theorem we get the convergence of the integrals, and the conclusion follows immediately.

Lemma 3. Let $s \in\left(1, \frac{3}{2}\right)$ and $u \in H^{s}\left(\mathbb{R}^{n}\right)$. Then $\Phi_{u}^{s} \in L^{1}\left(\mathbb{R}^{n} \times \mathbb{R}^{n}\right)$.

Proof. Take a sequence of functions $u_{h} \in \mathcal{C}_{0}^{\infty}\left(\mathbb{R}^{n}\right)$ such that $u_{h} \rightarrow u$ in $H^{s}\left(\mathbb{R}^{n}\right)$ and almost everywhere. Since $\Phi_{u_{h}}^{s} \rightarrow \Phi_{u}^{s}$ a.e. on $\mathbb{R}^{n} \times \mathbb{R}^{n}$, Fatou's Lemma, Lemma 2 for $u_{h}$ and the boundeness of $v \mapsto v^{ \pm}$in $H^{s}\left(\mathbb{R}^{n}\right)$ give

$$
\begin{aligned}
\iint_{\mathbb{R}^{n} \times \mathbb{R}^{n}} \Phi_{u}^{s}(x, y) d x d y & \leq \liminf _{h \rightarrow \infty} \iint_{\mathbb{R}^{n} \times \mathbb{R}^{n}} \Phi_{u_{h}}^{s}(x, y) d x d y \\
& =c(n, s) \liminf _{h \rightarrow \infty}\left\langle\left(-\Delta_{\mathbb{R}^{n}}\right)^{s} u_{h}^{+}, u_{h}^{-}\right\rangle \\
& \leq c(n, s) \lim _{h \rightarrow \infty}\left\langle\left(-\Delta_{\mathbb{R}^{n}}\right)^{s} u_{h}, u_{h}\right\rangle=c(n, s)\left\langle\left(-\Delta_{\mathbb{R}^{n}}\right)^{s} u, u\right\rangle,
\end{aligned}
$$

that concludes the proof.

Proof of Theorem 1] Take a sequence $u_{h} \in \mathcal{C}_{0}^{\infty}\left(\mathbb{R}^{n}\right)$ such that $u_{h} \rightarrow u$ in $H^{s}\left(\mathbb{R}^{n}\right)$ and almost everywhere. Consider the nonnegative functions

$$
v_{h}:=u_{h}^{+} \wedge u^{+}=u^{+}-\left(u^{+}-u_{h}^{+}\right)^{+}, \quad w_{h}:=u_{h}^{-} \wedge u^{-}=u^{-}-\left(u^{-}-u_{h}^{-}\right)^{+} .
$$

Then $v_{h}, w_{h} \in H^{s}\left(\mathbb{R}^{n}\right)$. Next, take any exponent $\tau \in(1, s)$. By Proposition 1 we have that $u^{ \pm}-u_{h}^{ \pm} \rightarrow 0$ in $H^{\tau}\left(\mathbb{R}^{n}\right)$; hence $\left(u^{ \pm}-u_{h}^{ \pm}\right)^{+} \rightarrow 0$ in $H^{\tau}\left(\mathbb{R}^{n}\right)$ by Item 3 in 
the Introduction. Thus,

$v_{h} \rightarrow u^{+}, \quad w_{h} \rightarrow u^{-} \quad$ in $H^{\tau}\left(\mathbb{R}^{n}\right)$ and almost everywhere, as $h \rightarrow \infty$.

Now we take a small $\varepsilon>0$. Recall that $\left(v_{h}-\varepsilon\right)^{+} \in H^{\tau}\left(\mathbb{R}^{n}\right)$ by Lemma 1 . Moreover, from $0 \leq v_{h} \leq u_{h}^{+}, 0 \leq w_{h} \leq u_{h}^{-}$it follows that

$$
\operatorname{supp}\left(\left(v_{h}-\varepsilon\right)^{+}\right) \subseteq\left\{u_{h} \geq \varepsilon\right\} ; \quad \operatorname{supp}\left(w_{h}\right) \subseteq \operatorname{supp}\left(u_{h}^{-}\right) .
$$

In particular, the functions $\left(v_{h}-\varepsilon\right)^{+}, w_{h}$ have compact and disjoint supports. Thus we can apply Theorem 2 to infer

$$
\left\langle\left(-\Delta_{\mathbb{R}^{n}}\right)^{\tau}\left(v_{h}-\varepsilon\right)^{+}, w_{h}\right\rangle=-C_{n, \tau} \iint_{\mathbb{R}^{n} \times \mathbb{R}^{n}} \frac{\left(v_{h}(x)-\varepsilon\right)^{+} w_{h}(y)}{|x-y|^{n+2 \tau}} d x d y .
$$

We first take the limit as $\varepsilon \searrow 0$. The argument in the proof of Lemma 2 gives

$$
\left\langle\left(-\Delta_{\mathbb{R}^{n}}\right)^{\tau} v_{h}, w_{h}\right\rangle=-C_{n, \tau} \iint_{\mathbb{R}^{n} \times \mathbb{R}^{n}} \frac{v_{h}(x) w_{h}(y)}{|x-y|^{n+2 \tau}} d x d y .
$$

Next we push $h \rightarrow \infty$. By (8) we get

$$
\lim _{h \rightarrow \infty}\left\langle\left(-\Delta_{\mathbb{R}^{n}}\right)^{\tau} v_{h}, w_{h}\right\rangle=\left\langle\left(-\Delta_{\mathbb{R}^{n}}\right)^{\tau} u^{+}, u^{-}\right\rangle .
$$

Further, since the integrand in the right-hand side of (9) does not exceed $\Phi_{u}^{\tau}(x, y)$, Lemma 3, (8) and Lebesgue's theorem give

$$
\lim _{h \rightarrow \infty} \iint_{\mathbb{R}^{n} \times \mathbb{R}^{n}} \frac{v_{h}(x) w_{h}(y)}{|x-y|^{n+2 \tau}} d x d y=\iint_{\mathbb{R}^{n} \times \mathbb{R}^{n}} \Phi_{u}^{\tau}(x, y) d x d y .
$$

Thus, we proved (4) with $s$ replaced by $\tau$. It remains to pass to the limit as $\tau \nearrow s$. By Lebesgue's theorem, we have

$$
\begin{aligned}
\lim _{\tau \nearrow^{s}}\left\langle\left(-\Delta_{\mathbb{R}^{n}}\right)^{\tau} u^{+}, u^{-}\right\rangle & =\lim _{\tau \nearrow^{s}} \int_{\mathbb{R}^{n}}|\xi|^{2 \tau} \mathcal{F}\left[u^{+}\right] \overline{\mathcal{F}\left[u^{-}\right]} d \xi \\
& =\int_{\mathbb{R}^{n}}|\xi|^{2 s} \mathcal{F}\left[u^{+}\right] \overline{\mathcal{F}\left[u^{-}\right]} d \xi=\left\langle\left(-\Delta_{\mathbb{R}^{n}}\right)^{s} u^{+}, u^{-}\right\rangle .
\end{aligned}
$$

Now, we fix $\tau_{0} \in(1, s)$ and note that $0 \leq \Phi_{u}^{\tau} \leq \max \left\{\Phi_{u}^{\tau_{0}}, \Phi_{u}^{s}\right\}$ for any $\tau \in\left(\tau_{0}, s\right)$. Therefore, Lemma 3 and Lebesgue's theorem give

$$
\lim _{\tau \nearrow s} \iint_{\mathbb{R}^{n} \times \mathbb{R}^{n}} \Phi_{u}^{\tau}(x, y) d x d y=\iint_{\mathbb{R}^{n} \times \mathbb{R}^{n}} \Phi_{u}^{s}(x, y) d x d y .
$$

The proof of (4) is complete. The last statement follows immediately from (4), polarization identity and (2).

Example 1. It is easy to construct a function $u \in \mathcal{C}_{0}^{\infty}\left(\mathbb{R}^{n}\right)$ such that $u^{+} \in H^{s}\left(\mathbb{R}^{n}\right)$ if and only if $s<\frac{3}{2}$.

Take $\varphi \in \mathcal{C}_{0}^{\infty}(\mathbb{R})$ satisfying $\varphi(0)=0, \varphi^{\prime}(0)>0$ and $x \varphi(x) \geq 0$ on $\mathbb{R}$. By direct computation one checks that $\varphi^{+}=\chi_{(0, \infty)} \varphi \in H^{s}(\mathbb{R})$ if and only if $s<\frac{3}{2}$. If $n=1$ we are done. If $n \geq 2$ we take $u\left(x_{1}, x_{2}, \ldots, x_{n}\right)=\varphi\left(x_{1}\right) \varphi\left(x_{2}\right) \ldots \varphi\left(x_{n}\right)$. 


\section{Acknowledgments}

The first author wishes to thank Université Libre de Bruxelles for the hospitality in February 2016. She is grateful to Denis Bonheure, Nicola Abatangelo, Sven Jahros and Albero Saldaña for valuable discussion on this subject.

First author was Partially supported by Miur-PRIN 2015233N54. Second author was Supported by RFBR Grant 17-01-00678.

\section{References}

[1] N. Abatangelo, S. Jarohs and A. Saldaña, On the maximum principle for higher-order fractional Laplacians, preprint (2016), arxiv:1607.00929.

[2] G. Bourdaud and Y. Meyer, Fonctions qui opèrent sur les espaces de Sobolev, J. Funct. Anal. 97(2) (1991) 351-360.

[3] G. Bourdaud and W. Sickel, Composition operators on function spaces with fractional order of smoothness, Harmonic Analysis and Nonlinear Partial Differential Equations, RIMS Kokyuroku Bessatsu, B26, Res. Inst. Math. Sci. (RIMS), Kyoto, 2011, pp. 93132.

[4] R. Musina and A. I. Nazarov, On the Sobolev and Hardy constants for the fractional Navier Laplacian, Nonlinear Anal. 121 (2015) 123-129. Online version, http:// www.sciencedirect.com/science/article/pii/S0362546X14003113.

[5] A. I. Nazarov, Remark on fractional Laplacians, Preprints of St. Petersburg Mathematical Society 2016-01 (2016), Online: www.mathsoc.spb.ru/preprint/2016/index.html.

[6] P. Oswald, On the boundedness of the mapping $f \rightarrow|f|$ in Besov spaces, Comment. Math. Univ. Carolin. 33(1) (1992) 57-66.

[7] T. Runst and W. Sickel, Sobolev Spaces of Fractional Order, Nemytskij Operators, and Nonlinear Partial Differential Equations, de Gruyter Series in Nonlinear Analysis and Applications, Vol. 3 (de Gruyter, Berlin, 1996).

[8] H. Triebel, Interpolation Theory, Function Spaces, Differential Operators (DeutscherVerlag, Wissensch., Berlin, 1978). 